\title{
Increased injury and intramuscular collagen of the diaphragm in COPD: autopsy observations
}

\author{
A. Scott*, X. Wang*, J.D. Road ${ }^{*}$ and W.D. Reid**;
}

ABSTRACT: Evidence for diaphragm injury in people with chronic obstructive pulmonary disease (COPD) has been reported, although the extent of injury and collagen accumulation post mortem have not previously been examined. In addition, it is not known whether the amount of injury and collagen are different in key regions of the diaphragm.

The cross-sectional area of collagen and the percentage of abnormal myofibres in the post mortem diaphragm and psoas major were determined by computer-assisted image analysis of stained cross-sections of the diaphragm for collagen with picrosirius red and with haematoxylin and eosin for morphology.

In the midcostal diaphragm of six subjects with COPD and six subjects with no significant respiratory disease, the COPD diaphragm displayed a greater cross-sectional area of collagen and percentage of abnormal myofibres (collagen: $24.2 \pm 1.0$ versus $18.6 \pm 1.1 \%$; injury: $28.4 \pm 7.2$ versus $12.0 \pm 1.3 \%$ ). In 18 patients with various respiratory conditions, the midcostal diaphragm displayed more collagen and abnormal myofibres than the crural diaphragm, while both costal and crural diaphragms displaying more collagen and abnormal myofibres than psoas major.

This study reveals extensive injury and collagen accumulation in the chronic obstructive pulmonary diseased diaphragm, and reveals a regional pattern of injury and intramuscular collagen which may correspond to variations in diaphragm loading.

KEYWORDS: Aged, diaphragm, human, lung diseases, obstructive, pathology/physiopathology

$\mathbf{T}$ he generation of inspiratory pressure by the diaphragm involves the transmission of forces generated by the myofibres across the collagens of the extracellular matrix $[1,2]$. The collagen reinforcements within skeletal muscles include the basal lamina, endomysium and perimysium, all of which are involved in active and passive force transmission [3]. In limb muscles, the amount of collagen in these structures can increase in response to exertioninduced muscle injury [4, 5]. In particular, chronic exertional muscle injury is associated with repeated injury and disruption of regenerating myotubes, a long-term decrease in the relative cross-sectional area of contractile tissue, and the accumulation of intramuscular collagen $[5,6]$.

Many patients with chronic obstructive pulmonary disease (COPD) show high levels of diaphragm activity both at rest and during exercise [7]. It is likely that during repeated or prolonged exacerbations, the COPD diaphragm may experience maximal activation leading to injury and incomplete regeneration, as shown in animal models [8-11]. Although individuals with COPD show a high susceptibility to exertioninduced diaphragm injury following inspiratory resistive loading [12], the extent of injury has, to date, been examined only in stable COPD patients. It is not known what extent of injury would be present in COPD patients post mortem, many of whom die in respiratory distress during periods of increased diaphragm loading.

The main objective of this study was to determine whether individuals with COPD have a greater area fraction of intramuscular collagen and proportion of injured myofibres in the diaphragm compared with controls; subjects with no significant respiratory disease. The authors hypothesised that the diaphragm of individuals with COPD would have a greater cross-sectional area of collagen and a higher percentage of injured or abnormal myofibres. It was further hypothesised that the costal diaphragm would display more injury and collagen accumulation than the crural, and that a nonrespiratory muscle (psoas major)

\section{AFFILIATIONS}

*School of Rehabilitation Sciences, ${ }^{\#}$ Respiratory Division and "James Hogg iCAPTURE Centre for Cardiovascular and Pulmonary Research, University of British Columbia, BC, Canada.

CORRESPONDENCE

W.D. Reid

School of Rehabilitation Sciences T325-2211 Wesbrook Mall

Vancouver

BC V6T 2B5 Canada

Fax: 16048227624

E-mail: wdreid@interchange.ubc.ca

Received:

December 142004

Accepted after revision:

October 042005

\section{SUPPORT STATEMENT}

This study was supported with funding from the British Columbia Lung Association and British Columbia Medical Services Foundation. 
would show minimal injury and a lower cross-sectional area of collagen, corresponding to the relative levels of activation and injury in each of these muscles.

\section{METHODS}

\section{Study subjects}

Ethical approval was obtained from the review board for human studies at the University of British Columbia, Vancouver Hospital and Health Sciences Centre and St Paul's Hospital (Vancouver, BC, Canada). The study was consistent with the Helsinki Declaration of 1975, as revised in 2000. Consent was obtained from next of kin. Subjects with the following conditions were excluded: death $>96 \mathrm{~h}$ prior to the autopsy, Hepatitis B and C, HIV, congestive heart failure, neuromuscular disease and myopathy, and corticosteroid doses $>20 \mathrm{mg}$ daily. Autopsy findings and hospital charts were reviewed by a respirologist. COPD subjects had more than two of the following: a computerised axial tomography scan showing emphysema; a reduced forced expiratory volume in one second (FEV1)/forced vital capacity ratio $<60 \%$; a smoking history $>20 \mathrm{yrs}$; or a history of breathlessness or chronic cough. Significant restrictive defects (kyphoscoliosis or other chest wall deformities) were absent on chest radiographs. Inclusion criterion for controls was a lack of respiratory pathology in the history and at autopsy. For the regional study, samples were taken from consecutive subjects undergoing autopsies at two teaching hospitals. Exclusion criteria were legal proceedings, Hepatitis B or C, HIV, or death $>96 \mathrm{~h}$ prior to the autopsy.

\section{Biopsy sampling and histology}

For comparison between COPD and control subjects, the right or left midcostal diaphragm was sampled. For regional studies, whole diaphragms were removed and pinned in anatomic orientation on paraffin wax in formalin (fig. 1). A biopsy was taken from the psoas major, midway along its length. Biopsy $6-\mu \mathrm{m}$ sections were embedded in paraffin and stained for

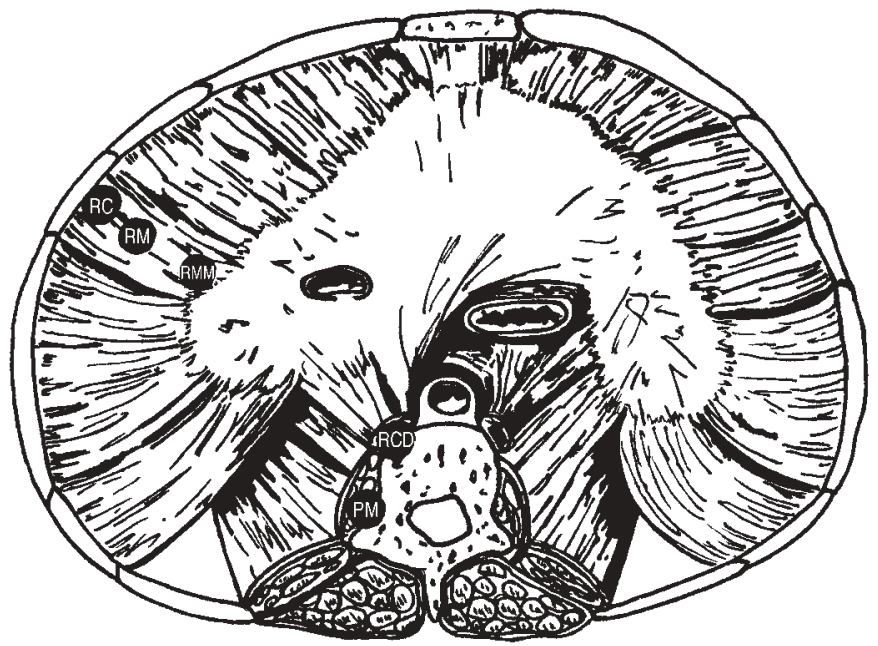

FIGURE 1. Diaphragm sample sites. RC: right costal insertion (as close as possible to the costal insertion in the midaxillary line); RM: right midcostal; RMM: right musculotendinous midaxillary insertion ( $\sim 3 \mathrm{~mm}$ from the visible beginning of the central tendon in the midaxillary line); PM: psoas major muscle belly; RCD: right crural diaphragm midway between origin and insertion. collagens (picrosirius red) or morphology (haematoxylin and eosin: H\&E). The picrosirius red stain was selected because it specifically labels collagen molecules without relying on the recognition of antigens, which may be degraded post mortem or during fixation [13]. A pilot study of an autolytic change in rat diaphragms demonstrated that up to $96 \mathrm{~h}$ the only post mortem morphological artefact was a decrease in the intensity of H\&E staining [14]. This artefact was not observed in the current study.

\section{Quantification of collagen and abnormal myofibres}

Fifteen randomly selected viewing fields per picrosirius red section were digitised by a blinded investigator using a Nikon brightfield microscope (Nikon Canada Inc, Mississauga, ON Canada) and SPOT digital camera (Diagnostic Instruments, Sterling Heights, MI, USA) avoiding large vessels and tissue edges. The collagen cross-sectional area was determined by counting a 63-point grid using calibrated software (Image Pro Plus, Media Cybernetics, Silver Spring, MD, USA), with an inter-rater reliability of $\mathrm{r}=0.957$.

A total of 20 randomly selected viewing fields per H\&E section were digitised and myofibres were categorised to determine the percentage of myofibres with abnormal morphology. Abnormal morphology was defined using predetermined categories (table 1). Inter-rater reliability of the categories ranged from $r=0.94$ to $r=0.96$. To determine the myofibre cross-sectional area, >200 myofibres per biopsy were outlined in Image Pro Plus. Inter-rater reliability of measures for myofibre cross-sectional area was $\mathrm{r}=0.99$ based on 100 independently measured myofibres. Diaphragm thickness was quantified using images digitised at $\times 20$ magnification by measuring the distance between abdominal and thoracic surfaces at six to eight equal intervals along the length of each cross-section.

\section{Statistical analysis}

To compare age- and sex-matched COPD with control groups, paired $t$-tests were used $(p<0.05)$. To test for regional

\begin{tabular}{|c|c|c|}
\hline TABLE 1 & \multicolumn{2}{|c|}{$\begin{array}{l}\text { Categories for computerised point counting of } \\
\text { haematoxylin and eosin stained cross-sections } \\
\text { of the diaphragm }\end{array}$} \\
\hline Features & & Definition \\
\hline \multicolumn{3}{|l|}{ Normal } \\
\hline \multicolumn{2}{|c|}{ Peripheral nucleus } & Nucleus situated within $2 \mu \mathrm{m}$ of sarcolemma \\
\hline \multicolumn{2}{|c|}{ Normal cytoplasm } & Evenly textured, eosinophilic cytoplasm \\
\hline \multicolumn{2}{|c|}{ Normal size and shape } & Homogenous size, polygonal shape \\
\hline \multicolumn{3}{|c|}{ Abnormal } \\
\hline \multicolumn{2}{|c|}{ Internal nucleus } & $\begin{array}{l}\text { Minimum } 2 \mu \mathrm{m} \text { of cytoplasm between } \\
\text { nucleus and sarcolemma }\end{array}$ \\
\hline \multicolumn{2}{|c|}{ Abnormal cytoplasm } & $\begin{array}{l}\text { Pale staining or basophilic cytoplasm; granular, } \\
\text { whorled, vacuolated or disrupted texture; loss of } \\
\text { sarcolemma integrity; lipofuscin accumulation; } \\
\text { rounded, deeply eosinophilic fibre }\end{array}$ \\
\hline \multicolumn{2}{|c|}{ Abnormal size or shape } & $\begin{array}{l}\text { Small fibre }(<1 / 3 \text { diameter of three largest fibres } \\
\text { in the field), angulated fibre with concave edges, } \\
\text { lobulated fibre }\end{array}$ \\
\hline
\end{tabular}


differences within the costal diaphragm and between the costal and crural diaphragm and psoas, ANOVA was used. To determine group differences in collagen cross-sectional areas, 15 fields from each subject were pooled [15] for analysis by group and region. For significant main effects, Tukey's post hoc test was used. To test whether the COPD and control myofibre size distributions were different, the Kolmogorov-Smirnov test was used [16].

\section{RESULTS}

\section{COPD versus control}

From the 77 biopsies gathered in the larger post mortem study undertaken, 12 met the inclusion and exclusion criteria for the COPD and control groups, and could be age- and sex-matched (table 2). All of the COPD subjects had an additional respiratory diagnosis, while none of the control subjects showed any evidence of respiratory pathology. Five of the six COPD patients had respiratory related deaths, while four of the six control subjects had cardiac-related deaths (table 2). One COPD patient experienced prolonged mechanical ventilation (35 days), with repeated attempts to wean, followed by 14 days on an assisted-controlled ventilator. The spirometric reports for five of the six COPD subjects showed a mean $\pm \mathrm{SE}$ FEV1 of $1.37 \pm 0.40 \mathrm{~L}$ (range: $0.80-1.91 \mathrm{~L} ; 33-52 \%$ predicted). Although FEV1 is a useful marker of disease progression, it does not correlate well with the degree of dyspnoea or mortality [17]; however the current authors report this measure as it was consistently available in a retrospective chart review.

In processed cross-sections of the diaphragm for picrosirius red, collagen was located in the endomysium, perimysium, epimysium, basal lamina and vessel walls. The only other structures that stained positively for collagen were nerve sheaths, which were not included in the point counting. Point counting revealed a greater cross-sectional area of collagen (mean $\pm \mathrm{SE}$ ) in biopsies from the COPD group compared with the controls ( $24.2 \pm 1.9$ versus $18.6 \pm 2.8 \%, p<0.001$; fig. 2 ). Focal areas of collagen accumulation associated with small myofibres or signs of necrosis were observed in the COPD diaphragm and, rarely, in the control group.

H\&E staining revealed a variety of abnormalities that were present in both COPD and control diaphragms, but were more extensive in the COPD biopsies. Examples from COPD patients are shown in figure 2. The COPD diaphragm had a higher percentage of abnormal myofibres ( $28.4 \pm 7.2$ versus $12.0 \pm 1.3 \%$; $\mathrm{p}<0.05)$ and sub-category analysis showed the most predominant feature was an abnormal cytoplasm (COPD: 16.0 $\pm 5.3 \%$; control: $2.5 \pm 1.5 \%$; p <0.05; fig. 3 ). Common observations of abnormal cytoplasm were lipofuscin accumulation; absent cytoplasm centrally or peripherally; and nonhomogeneous cytoplasm with distinct regions of peripheral basophilia, hyper-eosinophilia, and central or peripheral eosinophilia. In addition, large rounded myofibres with a glassy eosinophilic texture were also observed. The percentage of internally nucleated myofibres (COPD: $8.8 \pm 2.2$; control: $6.4 \pm 0.9$ ) and abnormally shaped myofibres (COPD: 3.6 \pm 0.9 ; control: $2.1 \pm 0.7$ ) were not significantly different (fig. 3).

The median diaphragm myofibre area for COPD patients was $973.7 \mu \mathrm{m}^{2}$, compared with $1,136.7 \mu \mathrm{m}^{2}$ for the control group and the Kolmogorov-Smirnov test showed their cumulative distributions to be different, with a greater proportion of smaller fibres in the COPD biopsies ( $\mathrm{p}<0.001$; fig. 4). Despite this difference, the mean fibre area of the COPD patient's diaphragm was not different from control values $(1,208$ versus $1,237 \mu^{2}$ ), and neither was there any difference in the thickness of the COPD and control diaphragms $(2.4 \pm 0.4$ versus $2.2 \pm 0.2 \mathrm{~mm})$.

\section{Regional differences in the diaphragm}

Subjects from whom whole diaphragms were obtained $(n=18)$ died of a variety of acute and chronic causes (table 3 ). Twothirds of these patients had an acute or chronic respiratory

TABLE 2 Clinical characteristics of subjects with chronic obstructive pulmonary disease (COPD) versus non-COPD

\begin{tabular}{|c|c|c|c|c|c|c|c|c|}
\hline Group & Age yrs & Sex & BMI & Cause of death & $\begin{array}{l}\text { Corticosteroids } \\
\mathrm{mg} \cdot \mathrm{day}^{-1}\end{array}$ & $\begin{array}{l}\text { Mechanical } \\
\text { ventilation } \mathrm{h}\end{array}$ & $\begin{array}{l}\text { Additional } \\
\text { respiratory } \\
\text { diagnoses }\end{array}$ & $\begin{array}{l}\text { FEV1 L \% } \\
\text { predicted }\end{array}$ \\
\hline \multirow[t]{5}{*}{ Non-COPD ${ }^{\#}$} & 62 & M & 27.3 & Cardiac arrest & $\mathrm{N}$ & $<24$ & $\mathrm{~N}$ & NA \\
\hline & 78 & M & 27.4 & Myocardial infarction & $\mathrm{N}$ & $N$ & $\mathrm{~N}$ & NA \\
\hline & 73 & M & 27.0 & Myocardial infarction & N & $\mathrm{N}$ & $\mathrm{N}$ & NA \\
\hline & & & & Cancer breast and & & & & \\
\hline & 60 & $\mathrm{~F}$ & 25.2 & liver & N & $\mathrm{N}$ & $\mathrm{N}$ & NA \\
\hline \multirow[t]{4}{*}{ COPD } & 69 & M & & Myocardial infarction & $\mathrm{N}$ & 24 & Pneumonia & $1.44,61$ \\
\hline & 78 & M & 30.3 & Respiratory failure & 10 & $<1$ & Pneumonia & $1.23,55$ \\
\hline & 75 & M & 25.1 & Respiratory failure & $\mathrm{N}$ & 48 & Pneumonia & $1.45,43$ \\
\hline & 55 & $\mathrm{~F}$ & 27.3 & Respiratory failure & 7.5 & 35 days & Pneumonia & $0.80,33$ \\
\hline
\end{tabular}

BMI: body mass index; FEV1: forced expiratory volume in one second; M: male; F: female: N; none: NA: not available. ${ }^{\#: ~ m e a n ~} \pm$ SE age: $65.8 \pm 9.6$; BMI: $24.1 \pm 4.6$.

: mean \pm SE age: $68.5 \pm 8.2$; BMl: $24.5 \pm 4.6$. 

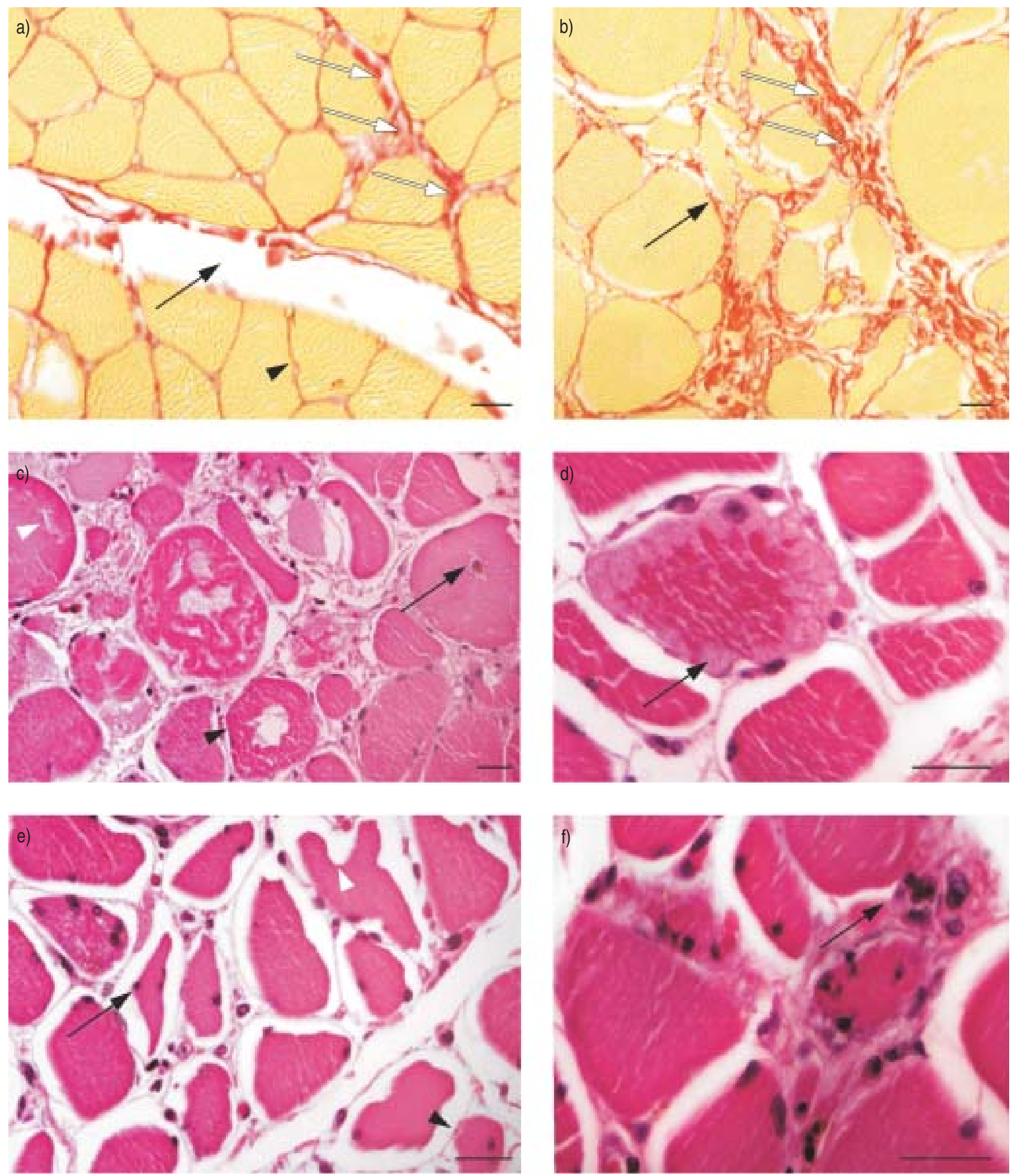

FIGURE 2. Photomicrographs of chronic obstructive pulmonary disease (COPD) diaphragm cross-sections stained with picrosirius red (a, b) or haemolysin and eosin (c-f). a) Normal distribution of endomysial (arrowhead) and perimysial collagen (white arrows). The black arrow points to separation artefact. b) Increased presence of collagen fibres in the endomysium (black arrow) and perimysium (white arrow). Note the small and abnormally shaped fibres embedded in the fibrotic region. c) Extensive injury in the midcostal diaphragm of a COPD subject. Black arrow shows lipofuschin accumulation, black arrowhead shows the disrupted cytoplasm and the white arrowhead shows the rounded fibre. d) Peripheral basophilia, enlarged nucleus. e) White arrowhead shows the lobulated fibre, the black arrow shows the angulated fibre and the black arrowhead shows the internally nucleated fibre. f) Increased cellularity and expanded interstitium. Scale bars $=20 \mu \mathrm{m}$. 

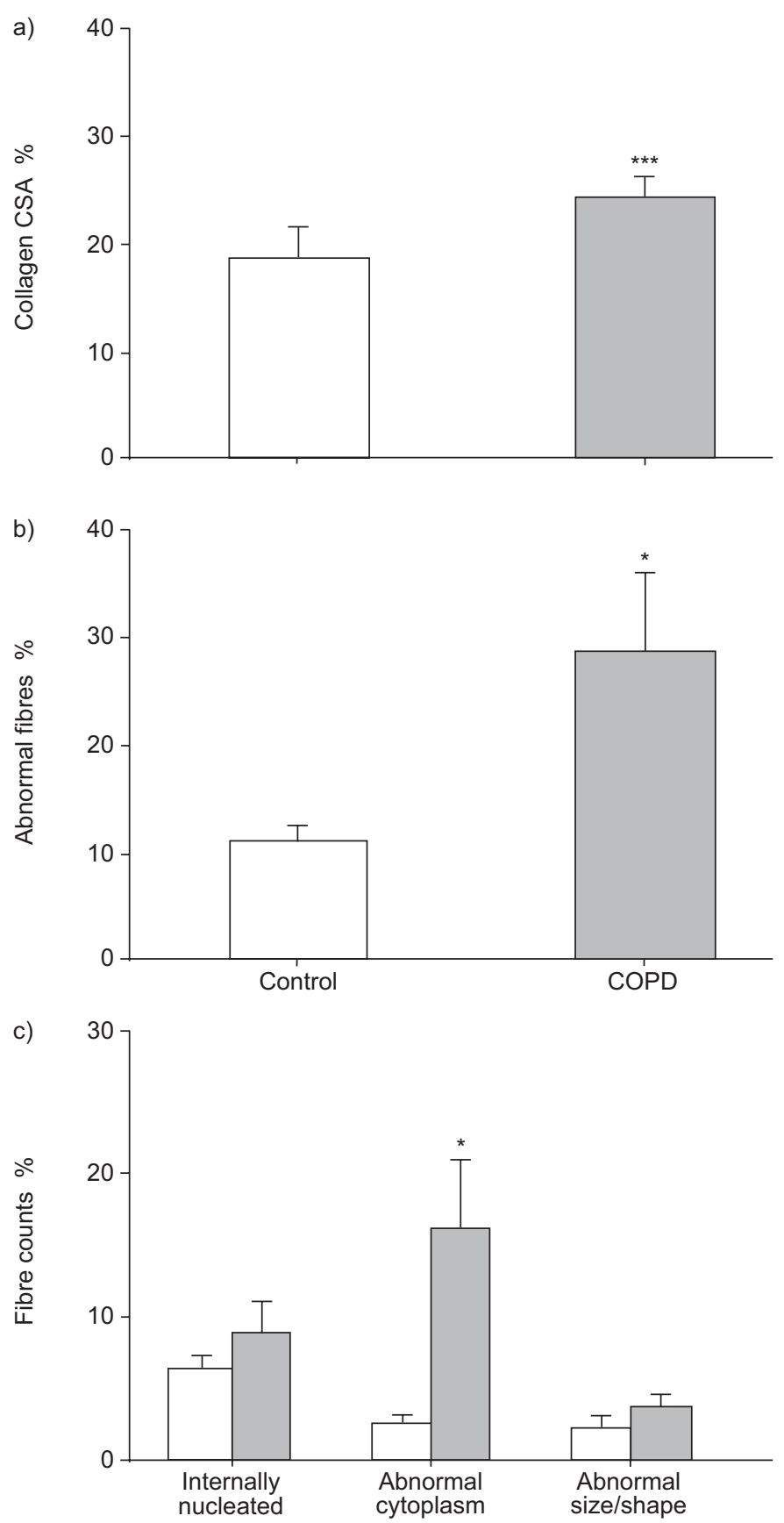

FIGURE 3. a) Cross-sectional area (CSA) of collagen in chronic obstructive pulmonary disease (COPD) and control subjects ( $n=6$ per group). b) Percentage of abnormal myofibres in COPD and control groups. c) Subcategories of abnormalities of muscle fibres in COPD and control subjects. ${ }^{*}: p<0.05$; ${ }^{* * *}: p<0.001$.

diagnosis. None of the regional study patients were mechanically ventilated for $>4$ days. Two regional study patients were taking corticosteroids, although the dose could not be determined from the medical records.

The localisation of collagen in all diaphragm regions was identical to that described above, with the exception that biopsies from the central tendon region showed areas stained

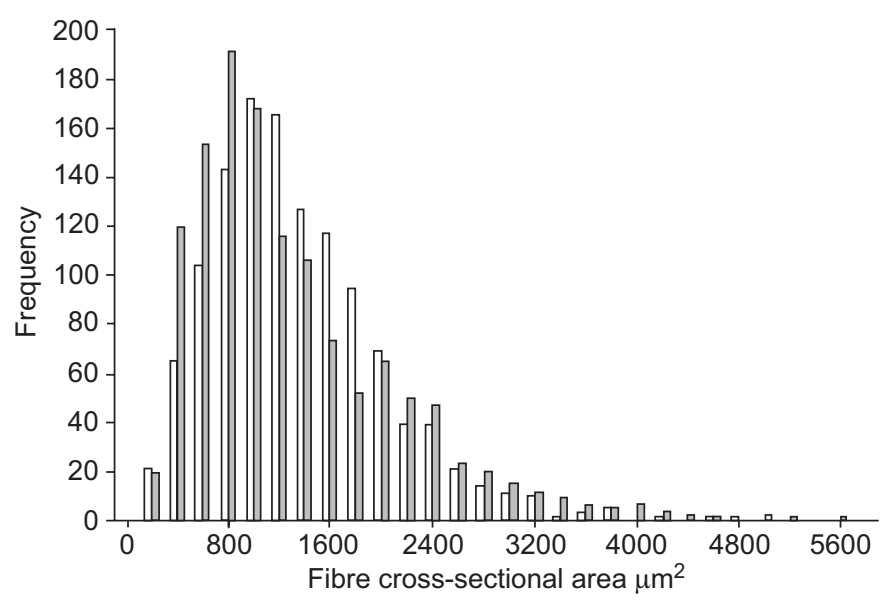

FIGURE 4. Cumulative distribution of myofibre cross-sectional areas in chronic obstructive pulmonary disease (COPD) patients ( $\square$ ) and control subjects ( $\square$ ). Note the increased proportion of small fibres in the COPD group.

densely red for collagen, which interdigitated between myofibres. The central tendon region had a higher collagen cross-sectional area than the costal insertion $(23.1 \pm 0.8$ versus $19.1 \pm 0.7 \%$; $<<0.01$; fig. $5 a$ ).

Abnormal diaphragm morphology was observed in all regions of the diaphragm examined. The percentage of abnormal myofibres in the midcostal region was higher than the costal insertion ( $14.6 \pm 2.6$ versus $11.8 \pm 2.3 \%$; $p<0.05)$, but lower than the central tendon region $(21.9 \pm 3.1 \%$; $<<0.05$; fig. $5 b)$. Analysis of subcategories revealed that the percentage of internally nucleated myofibres was highest in the central tendon region, a normal feature of the muscle-tendon junction region ( $p<0.01$; fig. 5c) [18]. The percentage of myofibres with abnormal size and shape in the midcostal diaphragm was higher than the costal insertion $(5.5 \pm 1.5$ versus $2.6 \pm 1.0 \%$, $\mathrm{p}<0.05$; fig. $5 \mathrm{c}$ ).

\section{Comparisons between psoas major and the costal and crural diaphragms}

The endomysium and perimysium in the psoas major appeared less dense and thick than in the midcostal or crural diaphragms, with a corresponding lower collagen crosssectional area than the midcostal and crural diaphragms (psoas: $12.3 \pm 0.5 \%$; crural: $18.0 \pm 0.6 \%$; midcostal: $20.9 \pm 0.6 \%$, $\mathrm{p}<0.01$; fig. 6a). The psoas major displayed less abnormal morphology, with few signs of necrosis or lipofuscin accumulation. The proportion of injured myofibres of psoas major was $8.3 \pm 2.2 \%$, which was lower than the midcostal and crural diaphragms $(p<0.01)$. The crural diaphragm had a lower proportion of injured myofibres than the midcostal $(11.9 \pm 2.5$ versus $14.6 \pm 2.6 \% ; \mathrm{p}<0.01$; fig. $6 \mathrm{~b})$. Analysis of subcategories showed that the psoas had less abnormal cytoplasm than the midcostal and crural diaphragms $(\mathrm{p}<0.05)$, and a tendency toward fewer myofibres with abnormal size and shape, than the midcostal diaphragm ( $p=0.065$; fig. $6 c)$. The midcostal diaphragm displayed more abnormally shaped myofibres than the crural diaphragm $(5.5 \pm 1.5$ versus $2.6 \pm 0.8 \% ; \mathrm{p}<0.01)$. The percentage of myofibres with abnormal cytoplasm in the 
TABLE 3 Clinical characteristics of the regional diaphragm study ${ }^{\#}$

\begin{tabular}{|c|c|c|c|c|c|c|}
\hline Age yrs & Sex & BMI & Cause of death & Corticosteroids mg day $^{-1}$ & Mechanical ventilation & Respiratory diagnoses \\
\hline 66 & $\mathrm{~F}$ & 30.8 & Respiratory failure & $\mathrm{N}$ & $\mathrm{N}$ & COPD, pulmonary oedema \\
\hline 62 & M & 27.3 & Cardiac arrest & $\mathrm{N}$ & $<24 \mathrm{~h}$ & N \\
\hline 78 & M & 30.3 & Respiratory failure & 10 & $<1 \mathrm{~h}$ & COPD, pneumonia \\
\hline 64 & M & 17.4 & Failed liver transplant & Y & 4 days & $\mathrm{N}$ \\
\hline 60 & $\mathrm{~F}$ & 25.2 & Cancer breast and liver & $\mathrm{N}$ & $\mathrm{N}$ & $\mathrm{N}$ \\
\hline 81 & M & 22.8 & Lymphoma & $\mathrm{N}$ & $\mathrm{N}$ & Pulmonary oedema \\
\hline 60 & M & 28.0 & Shock & Y past & $<48 \mathrm{~h}$ & Aspiration pneumonia \\
\hline 54 & M & 25.8 & Respiratory failure & $\mathrm{N}$ & 3 days & Bronchiolitis obliterans \\
\hline 78 & $\mathrm{~F}$ & 27.4 & Myocardial infarction & $\mathrm{N}$ & $\mathrm{N}$ & $\mathrm{N}$ \\
\hline 60 & M & 25.0 & Aortic aneurysm & N & $<1 \mathrm{~h}$ & $\mathrm{~N}$ \\
\hline 49 & M & 29.7 & Sepsis & Y & $48 \mathrm{~h}$ & Pneumonia, pleural effusion \\
\hline
\end{tabular}

BMI: body mass index; M: male; F: female; N: none; Y: yes; FEV1: forced expiratory volume in one second; COPD: chronic obstructive pulmonary disease. ${ }^{\#}$ : mean \pm SE age: $63.5 \pm 13.9$; BMI: $25.8 \pm 4.5$.

midcostal diaphragm $(8.4 \pm 2.9 \%)$ was higher than the crural diaphragm $(6.5 \pm 2.2 \% ; \mathrm{p}<0.05)$. There was no difference in the percentage of internally nucleated myofibres among the psoas and costal or crural diaphragms.

The proportion of abnormal fibres in the psoas major was not correlated with that of the midcostal diaphragm $\left(r^{2}=0.0859\right)$, indicating that patients with higher amounts of injury in the midcostal diaphragm did not display higher amounts of injury in a non-respiratory (control) muscle.

\section{DISCUSSION}

The COPD subjects in this study were experiencing an acuteon-chronic disease process, and the majority of COPD patients died of respiratory causes; thus, the present study presents novel observations of the COPD diaphragm following an acute-on-chronic increase in ventilatory loading. Sarcomeric disruptions have previously been shown in the COPD diaphragm at the electron microscope level in medically stable subjects; however, the diaphragm appeared normal under the light microscope with no evidence of inflammation or fibrosis [12]. In contrast, the current post mortem study demonstrates extensive injury and collagen accumulation (fibrosis) at the light microscope level. Fibrosis was associated with small, abnormally shaped myofibres (fig. 2b). Similar focal areas of fibrosis and small, misshaped myofibres have been reported in the diaphragm following chronic resistive loading in hamsters [10].

Whereas diaphragm fatigue is defined as a loss of forcegenerating capacity that is reversible by rest within hours or days, diaphragm injury is characterised by structural injury and a loss of force-generation with a more prolonged time course of recovery of several days or weeks, corresponding to the need to regenerate or repair the injury [19]. If exertional muscle injury is chronically repeated, regeneration may be compromised resulting in the pathological, long-term accumulation of collagen [5]. An association between collagen accumulation and injury of the human diaphragm has also been reported in the histopathology of sudden infant death syndrome [20]. Collagen is a major contributor to the passive mechanical properties of skeletal muscle, and also plays a role in the transmission of forces in the diaphragm, via linkages with the myofibrillar cytoskeleton through both integrin- $\beta 1$ complexes and dystrophin-dystroglycan complexes [1]. In avian muscular dystrophy, an increased amount of intramuscular connective tissue is associated with an increase in passive stiffness [21]. Thus the abnormal distribution and quantity of collagen observed in the COPD diaphragm may alter its mechanical properties and, potentially, the work of breathing.

The current authors found that the distributions of fibre size from the COPD and control groups were significantly different $(\mathrm{p}<0.001)$. The COPD diaphragm displayed a smaller median fibre area than in the control group $(973.7 \pm 22.9$ versus $\left.1,136.7 \pm 19.0 \mu \mathrm{m}^{2}\right)$, with an increased proportion of smaller myofibres (fig. 4). The COPD patients also displayed a greater number of extremely large myofibres; $2.9 \%$ of COPD myofibres were larger than $3,000 \mu^{2}$ in area, compared with $<1.0 \%$ in the controls. Despite these differences in myofibre size distribution, the mean myofibre cross-sectional areas were equivalent. This would argue against myofibre atrophy causing a relative increase in the collagen cross-sectional area.

It is likely that the injury and collagen accumulation that has been observed would lead to a loss of force-generation in the COPD diaphragm. A reduced volume of functioning myofibrils could be compensated by hypertrophy, or by an increased 

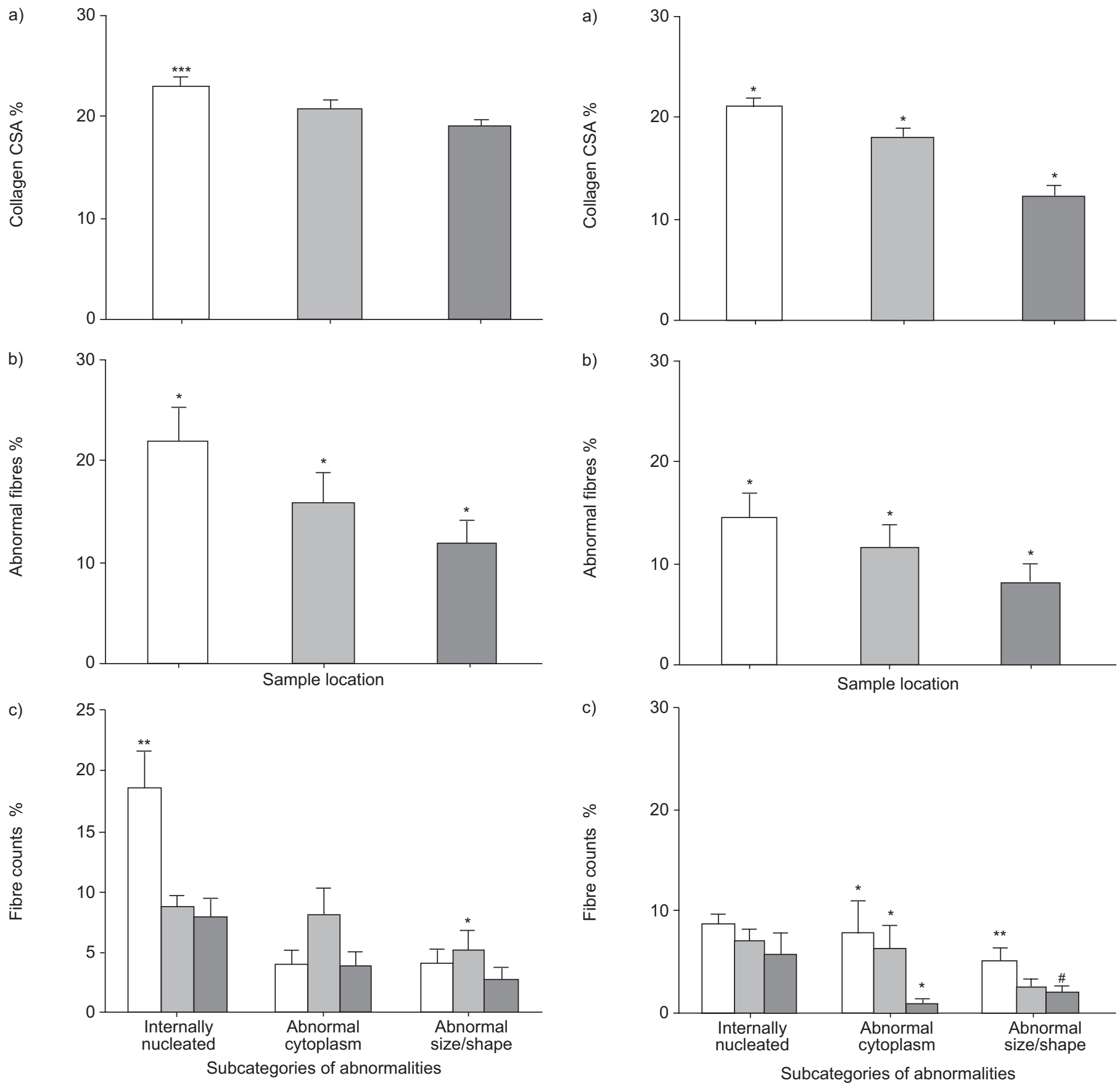

FIGURE 5. Cross-sectional area (CSA) of collagen (a) and percentage abnormal myofibres $(b, c)$ in different diaphragm regions ( $\square$ : central tendon region (CTR); $\square$ : midcostal; $\square$ : costal insertion. a) ${ }^{* \star *}: \mathrm{p}<0.001$, different from costal insertion. b) *: $p<0.05$, different from all other regions. $c) *: p<0.05$ different from costal insertion; ${ }^{*}: p<0.01$, different from all other regions for that category.

activation of the muscle. The mean fibre area was not different, which suggests that hypertrophy did not compensate significantly. Further, the diaphragm may already be maximally activated during acute exacerbations in some individuals with COPD [8]. In this case, the areas of disrupted sarcoplasm and collagen accumulation observed in this study may have contributed to a peri mortem loss of inspiratory force.

FIGURE 6. Cross-sectional area (CSA) of collagen (a) and the percentage of abnormal fibres (b, c) in the midcostal diaphragm $(\square)$, the crural diaphragm ( $\square$ ) and the psoas major $(\square){ }^{*}: p<0.05$, significant difference from each other region. ${ }^{\#}: p=0.065$, tendency to differ from midcostal; ${ }^{*}: p<0.01$ significant difference from crural; $n=18$

The current observations differ from those of LEVINE and coworkers $[22,23]$ in which surgical biopsies provided by stable COPD patients demonstrate not injury, but rather adaptive changes, characterised by fast-to-slow fibre-type transformations, as well as increases in mitochondrial volume fraction. This difference is likely to occur due to the stable patient sample in the cited studies, whereas patients in the 
current study samples were experiencing acute-on-chronic respiratory disease.

Although the amount of abnormal histology in the COPD diaphragm was statistically and significantly lower than the control diaphragm, the generalisability of this finding is limited by the relatively low number of subjects and inherent limitations of post mortem studies. The current authors have reported the FEV1, value, because it is a well validated index of disease progression, but the presence of expiratory flow limitation alone may not be the sole cause of the injury observed in this study. Dynamic lung volumes could not be assessed post mortem; it is likely that some patients presented with a mixed ventilatory impairment (i.e. obstructive plus restrictive ventilatory defects), especially due to the end-stage sequelae of COPD, including pneumonia and resultant subsegmental atelectasis. However, the current authors were able to rule out key factors other than COPD as the cause of injury. The COPD patients in this study did not display chestwall deformity on chest radiographs or post mortem, and were not in septic or cardiogenic shock. Only one COPD and one control patient had sepsis (which is known to increase sarcolemmal permeability and could contribute to diaphragm injury) [24]. Although one COPD patient had experienced prolonged mechanical ventilation (which is known to induce ultrastructural pathology), mechanical ventilation alone could not be the cause of the extensive light-microscopy changes observed in the current study.

The authors of the study were also limited in the ability to examine mechanistic or molecular features of interest such as expression levels of fibrogenic factors, or cytokines such as transforming growth factor- $\beta$ due to the delay between death and autopsy. Because the semi-quantitative assessment of collagen content could be influenced by the fixation and dehydration procedures, as well as the state of extracellular matrix hydration at the time of death, future studies should directly quantitate collagen or pro-collagen peptides.

The differences in injury and collagen between costal and crural diaphragms in this study are consistent with previous studies which have found distinct actions [25], timing [26] and fibre-type sizes and proportions [27] of these two regions. Models of diaphragm injury have revealed the costal diaphragm to be more susceptible to injury than the crural [28]. In the present study, the percentage of injured fibres was highest in the midcostal region. A study of regional diaphragm shortening in a canine model found that the midcostal region was the only region to experience eccentric contractions when the afterload was increased [29]. This supports the postulate that high levels of stress or strain may be contributing to injury in this region of the diaphragm.

The observation of a lower incidence of injury around the costal insertion has implications in the interpretation of previous studies of diaphragm injury, as this general region has been previously used as a surgical biopsy site [12]. Sampling from the costal insertion region in humans would appear not to represent the extent of injury in the midcostal region.

In the current study, the amount of injury and collagen in the diaphragm and the psoas major were compared. The collagen cross-sectional area and percentage of abnormal myofibres were highest in the costal diaphragm, followed by the crural diaphragm, with the lowest values in the psoas major. Like the diaphragm, the psoas major has a high percentage of type I muscle fibres (62\%) [30]. However, whereas the psoas can rest during times of illness and metabolic stress, the diaphragm must continue to contract, frequently to a greater degree if the work of breathing is increased. The high level of injury in the midcostal diaphragm highlights its susceptibility to exertioninduced injury in response to chronically increased loading.

\section{REFERENCES}

1 Boriek AM, Capetanaki Y, Hwang W, et al. Desmin integrates the three-dimensional mechanical properties of muscles. Am J Physiol Cell Physiol 2001; 280: C46-52.

2 Boriek AM, Zhu D, Zeller M, Rodarte JR. Inferences on force transmission from muscle fiber architecture of the canine diaphragm. Am J Physiol Regul Integr Comp Physiol 2001; 280: R156-165.

3 Huijing PA. Muscle as a collagen fiber reinforced composite: a review of force transmission in muscle and whole limb. J Biomech 1999; 32: 329-345.

4 Myllyla R, Salminen A, Peltonen L, Takala TE, Vihko V. Collagen metabolism of mouse skeletal muscle during the repair of exercise injuries. Pflugers Arch 1986; 407: 64-70.

5 Stauber WT, Smith CA, Miller GR, Stauber FD. Recovery from 6 weeks of repeated strain injury to rat soleus muscles. Muscle Nerve 2000; 23: 1819-1825.

6 Millems ME, Stauber WT. Force deficits after repeated stretches of activated skeletal muscles in female and male rats. Acta Physiol Scand 2001; 172: 63-67.

7 Sinderby C, Beck J, Spahija J, Weinberg J, Grassino A. Voluntary activation of the human diaphragm in health and disease. J Appl Physiol 1998; 85: 2146-2158.

8 Topeli A, Laghi F, Tobin MJ. The voluntary drive to breathe is not decreased in hypercapnic patients with severe COPD. Eur Respir J 2001; 18: 53-60.

9 Jiang TX, Reid WD, Belcastro A, Road JD. Load dependence of secondary diaphragm inflammation and injury after acute inspiratory loading. Am J Respir Crit Care Med 1998; 157: 230-236.

10 Reid WD, Belcastro AN. Chronic resistive loading induces diaphragm injury and ventilatory failure in the hamster. Respir Physiol 1999; 118: 203-218.

11 Machiels H, Verheul A, Croes H, Hafmans T, Dekhuijzen P. Ultrastructural changes in the diaphragm of aged emphysematous hamster. Basic Appl Myol 2002; 12: 201-208.

12 Orozco-Levi M, Lloreta J, Minguella J, Serrano S, Broquetas JM, Gea J. Injury of the human diaphragm associated with exertion and chronic obstructive pulmonary disease. Am J Respir Crit Care Med 2001; 164: 1734-1739.

13 Junquiera LC, Brentani RR. A simple and sensitive method for the quantitative estimation of collagen. Anal Biochem 1979; 94: 96-99.

14 Macgowan NA. Diaphragm injury in chronic respiratory disease. MSc thesis. Rehabilitation Sciences, University of British Columbia, Vancouver, Canada. 1998.

15 Anderson JE, Garret K, Moor A, McIntosh L, Penner K. Dystrophy and myogenesis in $\mathrm{mdx}$ diaphragm muscle. Muscle Nerve 1998; 21: 1153-1165. 
16 Conover WJ. Practical Nonparametric Statistics. New York, John Wiley and Sons, 1971.

17 Celli BR, Cate CG, Marin JM, Casanova C, Montes de Oca M. The body-mass index, airflow obstruction, dyspnea, and exercise capacity in chronic obstructive pulmonary disease. N Engl J Med 2004; 350: 1005-1012.

18 Anderson JR. Atlas of Skeletal Muscle Pathology. Lancaster, MTP Press Ltd, 1985.

19 Wang XY, Jiang TX, Reid WD, Road J. Ventilatory muscle injury. In: Hamid Q, Shannon J, Martin JG, eds. Ventilatory Muscle Injury. Hamilton, BC Decker Inc, 2004.

20 Kariks J. Diaphragmatic muscle fibre necrosis in sids. Forensic Sci Int 1989; 43: 281-291.

21 Feit H, Kawai M, Mostafapour AS. The role of collagen crosslinking in the increased stiffness of avian dystrophic muscle. Muscle Nerve 1989; 12: 486-492.

22 Levine S, Kaiser L, Leferovich J, Tikunov B. Cellular adaptations in the diaphragm in chronic obstructive pulmonary disease. N Engl J Med 1997; 337: 1799-1806.

23 Levine S, Gregory C, Nguyen T, et al. Bioenergetic adaptation of individual human diaphragmatic myofibers to severe COPD. J Appl Physiol 2002; 92: 1205-1213.

24 Lin MC, Ebihara S, El Dwairi Q, et al. Diaphragm sarcolemmal injury is induced by sepsis and alleviated by nitric oxide synthase inhibition. Am J Respir Crit Care Med 1998; 158: 1656-1663.

25 Ward ME, Macklem PT. Kinematics of the chest wall. In: Rousson C, ed. The Thorax. 2nd Edn. New York, Marcel Dekker, 1995; pp. 515-533.

26 Easton PA, Fitting JW, Arnoux R, Guerraty A, Grassino AE. Costal and crural diaphragm function during $\mathrm{CO} 2$ rebreathing in awake dogs. J Appl Physiol 1993; 74: 1406-1418.

27 Sanchez J, Medrano G, Debesse B, Riquet M, Derenne JP. Muscle fibre types in costal and crural diaphragm in normal men and in patients with moderate chronic respiratory disease. Bull Eur Physiopathol Respir 1985; 21: 351-356.

28 Reid WD, Huang J, Bryson S, Walker DC, Belcastro AN. Diaphragm injury and myofibrillar structure induced by resistive loading. J Appl Physiol 1994; 76: 176-184.

29 Wakai Y, Leevers AM, Road JD. Regional diaphragm shortening measured by sonomicrometry. J Appl Physiol 1994; 77: 2791-2796.

30 Parkkola R, Alanen A, Kalimo H, Lillsunde I, Komu M, Kormano M. Mr relaxation times and fiber type predominance of the psoas and multifidus muscle. An autopsy study. Acta Radiol 1993; 34: 16-19. 